\title{
The First Maximum-light Ultraviolet Through Near-infrared Spectrum of a Type la Supernova ,
}

\section{Citation}

Foley, Ryan J., Markus Kromer, G. Howie Marion, Giuliano Pignata, Maximilian D. Stritzinger, Stefan Taubenberger, Peter Challis, et al. 2012. "THE FIRST MAXIMUM-LIGHT ULTRAVIOLET THROUGH NEAR-INFRARED SPECTRUM OF A TYPE la SUPERNOVA,." The Astrophysical Journal 753 (1): L5. https://doi.org/10.1088/2041-8205/753/1/L5.

\section{Permanent link}

http://nrs.harvard.edu/urn-3:HUL.InstRepos:41412199

\section{Terms of Use}

This article was downloaded from Harvard University's DASH repository, and is made available under the terms and conditions applicable to Other Posted Material, as set forth at http:// nrs.harvard.edu/urn-3:HUL.InstRepos:dash.current.terms-of-use\#LAA

\section{Share Your Story}

The Harvard community has made this article openly available. Please share how this access benefits you. Submit a story.

Accessibility 


\title{
THE FIRST MAXIMUM-LIGHT ULTRAVIOLET THROUGH NEAR-INFRARED SPECTRUM OF A TYPE Ia SUPERNOVA*, $\dagger$
}

\author{
Ryan J. Foley ${ }^{1,12}$, Markus Kromer ${ }^{2}$, G. Howie Marion ${ }^{1}$, Giuliano Pignata ${ }^{3}$, Maximilian D. Stritzinger ${ }^{4,5}$, \\ Stefan Taubenberger ${ }^{2}$, Peter Challis ${ }^{1}$, Alexei V. Filippenko ${ }^{6}$, Gastón Folatelli $^{7}$, Wolfgang Hillebrandt ${ }^{2}$, \\ Eric Y. HsiaO ${ }^{8}$, Robert P. Kirshner ${ }^{1}$, Weidong Li ${ }^{6,13}$, Nidia I. Morrell ${ }^{8}$, Friedrich K. RöPKe ${ }^{2,9}$, \\ Franco Ciaraldi-Schoolmann ${ }^{2}$, Ivo R. Seitenzahl ${ }^{2,9}$, JefFrey M. Silverman $^{6}$, Robert A. Simcoe ${ }^{10}$, Zachory K. Berta $^{1}$, \\ Kevin M. Ivarsen ${ }^{11}$, Elisabeth R. Newton ${ }^{1}$, Melissa C. Nysewander ${ }^{11}$, and Daniel E. Reichart ${ }^{11}$ \\ ${ }^{1}$ Harvard-Smithsonian Center for Astrophysics, 60 Garden Street, Cambridge, MA 02138, USA; rfoley @ cfa.harvard.edu \\ ${ }^{2}$ Max-Planck-Institut für Astrophysik, Karl-Schwarzschild-Strasse 1, D-85748 Garching bei München, Germany \\ ${ }^{3}$ Departamento de Ciencias Fisicas, Universidad Andres Bello, Avda. Republica 252, Santiago, Chile \\ ${ }^{4}$ Department of Physics and Astronomy, Aarhus University, Ny Munkegade, DK-8000 Aarhus C, Denmark \\ ${ }^{5}$ The Oskar Klein Centre, Department of Astronomy, Stockholm University, AlbaNova, 10691 Stockholm, Sweden \\ ${ }^{6}$ Department of Astronomy, University of California, Berkeley, CA 94720-3411, USA \\ ${ }^{7}$ Kavli Institute for the Physics and Mathematics of the Universe (Kavli IPMU, WPI), Todai Institutes for Advanced Study, \\ The University of Tokyo, Kashiwa 277-8583, Japan \\ ${ }^{8}$ Carnegie Observatories, Las Campanas Observatory, La Serena, Chile \\ ${ }^{9}$ Institut für Theoretische Physik und Astrophysik, Universität Würzburg, Am Hubland, D-97074 Würzburg, Germany \\ ${ }^{10}$ MIT-Kavli Institute for Astrophysics and Space Research, 77 Massachusetts Avenue, 37-664D Cambridge, MA 02139, USA \\ ${ }^{11}$ Department of Physics and Astronomy, University of North Carolina at Chapel Hill, Chapel Hill, NC 27599, USA \\ Received 2012 February 23; accepted 2012 May 1; published 2012 June 13
}

\begin{abstract}
We present the first maximum-light ultraviolet (UV) through near-infrared (NIR) Type Ia supernova (SN Ia) spectrum. This spectrum of SN 2011iv was obtained nearly simultaneously by the Hubble Space Telescope at UV/optical wavelengths and the Magellan Baade telescope at NIR wavelengths. These data provide the opportunity to examine the entire maximum-light SN Ia spectral energy distribution. Since the UV region of an SN Ia spectrum is extremely sensitive to the composition of the outer layers of the explosion, which are transparent at longer wavelengths, this unprecedented spectrum can provide strong constraints on the composition of the SN ejecta, and similarly the SN explosion and progenitor system. SN 2011iv is spectroscopically normal, but has a relatively fast decline $\left(\Delta m_{15}(B)=1.69 \pm 0.05 \mathrm{mag}\right)$. We compare SN 2011iv to other SNe Ia with UV spectra near maximum light and examine trends between UV spectral properties, light-curve shape, and ejecta velocity. We tentatively find that SNe with similar light-curve shapes but different ejecta velocities have similar UV spectra, while those with similar ejecta velocities but different light-curve shapes have very different UV spectra. Through a comparison with explosion models, we find that both a solar-metallicity W7 and a zero-metallicity delayed-detonation model provide a reasonable fit to the spectrum of SN 2011iv from the UV to the NIR.
\end{abstract}

Key words: supernovae: general - supernovae: individual (SN 2011iv)

Online-only material: color figures

\section{INTRODUCTION}

Type Ia supernovae ( $\mathrm{SNe}$ Ia) are the energetic end of $\mathrm{C} / \mathrm{O}$ white dwarfs in binary systems that reach temperatures and densities high enough to cause a runaway chain of nuclear reactions (for a review, see Hillebrandt \& Niemeyer 2000). Despite having collected observations of thousands of SNe Ia, we still do not know what progenitor systems produce $\mathrm{SNe}$ Ia and do not fully understand how the star explodes. Measurements of SNe Ia led to the discovery of the accelerating universe (Riess et al. 1998; Perlmutter et al. 1999), and observing SNe Ia is still one of the best ways to constrain cosmological parameters.

\footnotetext{
* Based on observations made with the NASA/ESA Hubble Space Telescope, obtained at the Space Telescope Science Institute, which is operated by the Association of Universities for Research in Astronomy, Inc., under NASA contract NAS 5-26555. These observations are associated with program GO-12592.

$\dagger$ This Letter includes data gathered with the $6.5 \mathrm{~m}$ Magellan Telescopes located at Las Campanas Observatory, Chile.

${ }^{12}$ Clay Fellow.

${ }^{13}$ Deceased 2011 December 12.
}

It behooves us to understand the physics behind SN Ia explosions, and with additional knowledge of the progenitors and explosions, we hope to further improve the utility of SNe Ia for measuring cosmic distances.

SN Ia UV spectra are dominated by a forest of overlapping lines from iron-group elements (IGEs). UV photons are repeatedly absorbed and re-emitted in those lines and gradually scattered redward, where lower opacities allow them to escape. The UV is crucial to the formation of the optical spectral energy distribution (SED) of SNe Ia (Sauer et al. 2008), and extremely sensitive to both the progenitor composition and explosion mechanism (Höflich et al. 1998; Lentz et al. 2001). Observations of the UV directly probe the composition of the outermost layers of ejecta, which are transparent at optical wavelengths soon after explosion.

After light-curve shape correction (e.g., Phillips 1993), the luminosity of an SN Ia still depends significantly on hostgalaxy environment (Kelly et al. 2010; Lampeitl et al. 2010; Sullivan et al. 2010). This may indicate that there are subtle environmental effects imprinted on the progenitor stars that directly affect our luminosity calibration. Different progenitor 
metallicity may affect the outcome of the explosion (Timmes et al. 2003), the relation between luminosity and light-curve shape (Howell et al. 2007; Mazzali et al. 2001; Mazzali \& Podsiadlowski 2006), and the observed UV spectrum, especially at $\lambda<2600 \AA$ (Höflich et al. 1998; Lentz et al. 2000).

At optical wavelengths, SNe Ia have remarkably uniform luminosity ( $\sigma \approx 0.16$ mag; e.g., Hicken et al. 2009), after correcting for light-curve shape and color. This relationship extends to the $U$ band, but with larger scatter (Jha et al. 2006). The scatter can be further reduced to 0.11 mag after making a correction based on a measurement of the ejecta velocity (Foley \& Kasen 2011; Foley et al. 2011; Foley 2012). The intrinsic $B-V$ color of SNe Ia correlates strongly with ejecta velocity, with redder SNe having higher velocity. This is explained as additional line blanketing in the $B$ band of the high-velocity $\mathrm{SNe}$, and this trend should extend to the UV (Foley \& Kasen 2011).

High-quality UV spectra for low- $z$ SNe Ia are of particular importance to the calibration of high- $z$ SNe Ia. Since the observed SN light originates as rest-frame UV, most $z>1 \mathrm{SNe}$ Ia only have rest-frame UV light curves, and low- $z$ UV spectra are critical for understanding these data. Our current lack of UV spectra limits $K$-corrections and even SN classification at $z>1$ (Riess et al. 2007).

$\mathrm{SNe}$ Ia bright enough for the International Ultraviolet Explorer were rare (Foley et al. 2008), and only one SN Ia has a published high signal-to-noise ratio $(\mathrm{S} / \mathrm{N})$ Hubble Space Telescope (HST) spectrum near maximum-light covering wavelengths $\lesssim 2900 \AA-$ SN 1992A (Kirshner et al. 1993, hereafter K93). Swift has obtained spectra of several SNe Ia, but except for a few SNe (e.g., SN 2009ig; Foley et al. 2012), the spectra are generally of such low $\mathrm{S} / \mathrm{N}$ that detailed spectral analysis is not feasible (Bufano et al. 2009). A Cycle 13 HST program (GO-10182; PI: Filippenko) attempted to observe several SNe Ia in the UV; unfortunately, the Space Telescope Imaging Spectrograph (STIS) failed before any data could be obtained. The program was executed using the Advanced Camera for Surveys prism, which does not provide sufficient resolution to distinguish spectral features (Wang et al. 2011). In Cycle 17, 30 SNe Ia were observed at a single epoch by HST (GO-11721; PI: Ellis); however, these spectra did not probe below $2900 \AA$ (Cooke et al. 2011). It has been 20 years since the last high-S/N true-UV maximum-light SN Ia spectrum has been observed and published.

SN 2011 iv was discovered at an unfiltered magnitude of 12.8 on 2011 December 2.57 (UT dates are used throughout) by Drescher \& Parker (2011). There was no object detected on 2011 October 29.57 to a limit of $18.8 \mathrm{mag}$. It was discovered in NGC 1404 ( $\sim 1.3 \mathrm{kpc}$ from the nucleus), an elliptical galaxy in the Fornax cluster at $c z=1947 \mathrm{~km} \mathrm{~s}^{-1}$ (Graham et al. 1998) that also hosted SN 2007on (e.g., Stritzinger et al. 2011b) and is at $D=20.4 \mathrm{Mpc}\left(\mu=31.53 \pm 0.07 \mathrm{mag}^{14}\right.$ from a surface brightness fluctuation measurement; Blakeslee et al. 2010).

Noguchi et al. (2011) and Stritzinger et al. (2011a) obtained optical spectra of SN 2011iv on 2011 December 3.7 and 4.1, respectively, and determined that it was a young $\mathrm{SN}$ Ia. We triggered multiple programs to study the photometric and spectroscopic evolution of the $\mathrm{SN}$, its circumstellar environment, its polarization, its energetics, and other aspects. In particular, we triggered our HST target-of-opportunity program to obtain UV

\footnotetext{
14 This measurement is consistent with the Blakeslee et al. (2010) weighted average distance modulus for the Fornax cluster of $\mu=31.54 \pm 0.02 \mathrm{mag}$, but is larger than other distance estimates that have higher uncertainty.
}

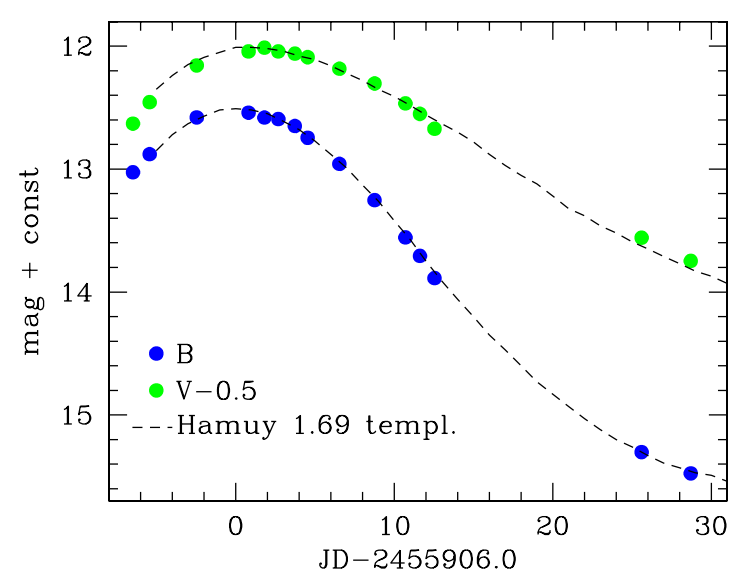

Figure 1. $B$ (blue points) and $V$ (green points) light curves of SN 2011iv. The dashed curves correspond to the $\Delta m_{15}=1.69$ mag Hamuy et al. (1996) light-curve templates.

(A color version of this figure is available in the online journal.)

spectra of SNe Ia (GO-12592; PI: Foley; Foley 2011). The first of seven UV spectra was obtained on 2011 December 11.08 and covered wavelengths of 1615-10230 ̊. Nearly simultaneously (2011 December 11.24), we obtained a near-infrared (NIR) spectrum with the Folded Port Infrared Echellette (FIRE) spectrograph (Simcoe et al. 2008) on the $6.5 \mathrm{~m}$ Magellan Baade telescope. In this Letter, we focus on this first UV-optical-NIR (UVOIR) spectrum spanning 0.16-2.5 $\mu \mathrm{m}$.

\section{OBSERVATIONS}

Optical photometry of SN 2011iv was collected with the Panchromatic Robotic Optical Monitoring and Polarimetry Telescopes (PROMPT) 3 and 5 (Reichart et al. 2005). Basic data reduction (bias and flat-field correction) was performed using standard routines in IRAF. Local standard stars were measured by Stritzinger et al. (2011b). The complex background around SN 2011iv was modeled with a low-order polynomial surface. Once the galaxy contamination was removed, photometry was performed using the point-spread-function fitting technique. We present our $B$ and $V$ light curves in Figure 1.

SN 2011 iv was observed by HST using the STIS spectrograph on 2011 December 11.08, corresponding to $t=0.6$ days relative to $B$ maximum (see Section 3 ). The observations were obtained over two orbits with three different gratings, all with the $52^{\prime \prime} \times 0$ ". 2 slit. Exposures of 2200 and $1350 \mathrm{~s}$ utilized the near-UV MAMA detector and the G230L grating. Two exposures of $100 \mathrm{~s}$ were taken with both the CCD/G430L and CCD/G750L setups, respectively. The three setups yield a combined wavelength range of 1615-10230 A.

The data were reduced using the standard HST Science Data Analysis System routines to bias subtract, flat-field, extract, wavelength-calibrate, and flux-calibrate each SN spectrum.

Almost immediately after obtaining the STIS spectrum (on 2011 December 11.24), we obtained an NIR spectrum $(0.83-2.5 \mu \mathrm{m})$ of SN 2011iv with FIRE. SN 2011iv was observed in the high-resolution echellette mode with the 0.6 slit, yielding a resolution of $R=6000$ or about $50 \mathrm{~km} \mathrm{~s}^{-1}$. Four frames were taken on source with 240 s exposures using ABBA nodding.

The data were reduced using a custom-developed IDL pipeline (FIREHOSE), which evolved from the MASE pipeline used for optical echelle reductions (Bochanski et al. 2009). The 


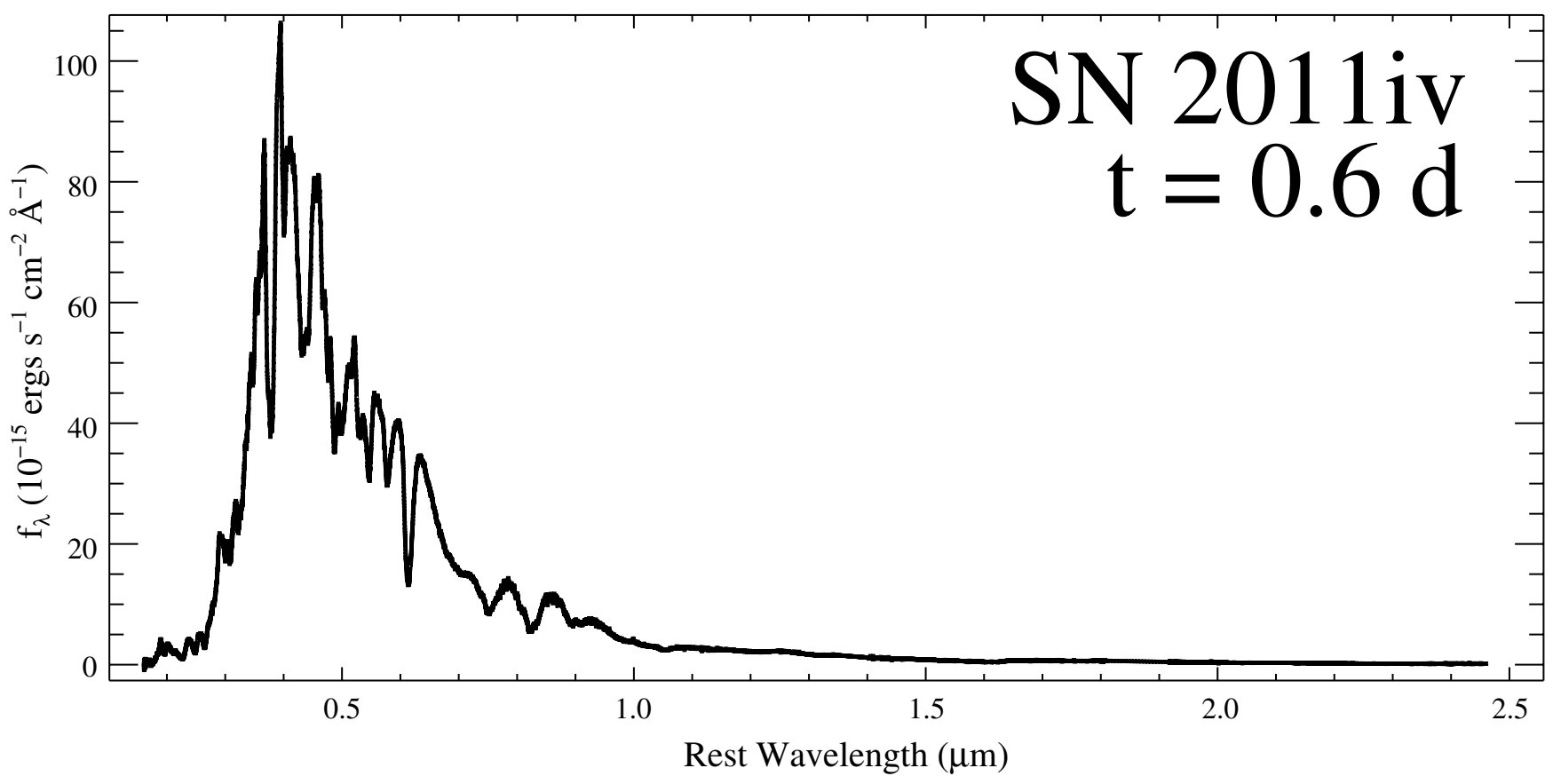

Figure 2. UVOIR HST/STIS and Magellan/FIRE maximum-light spectrum of SN 2011iv.

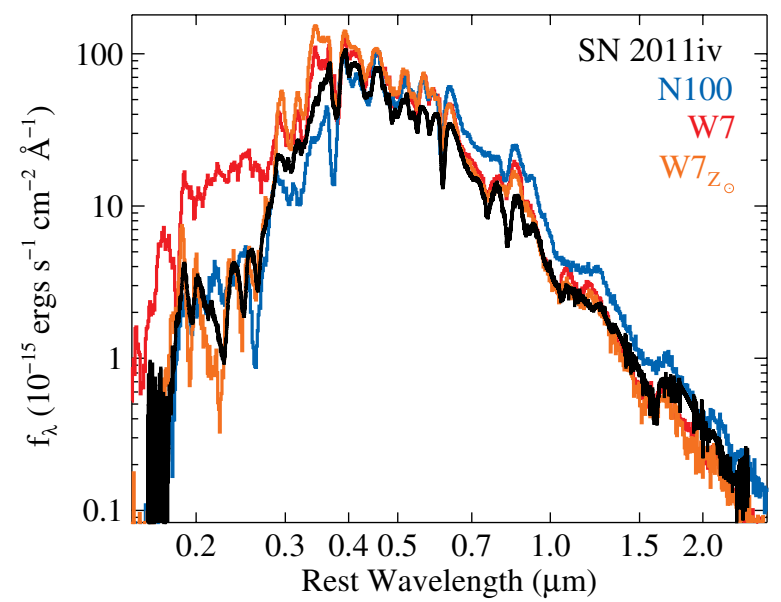

Figure 3. UVOIR maximum-light spectrum of SN 2011 iv (black curve). This spectrum is the same as that shown in Figure 2, but with a different scale to highlight the UV and NIR portions. The blue, red, and orange curves represent model spectra generated from a zero-metallicity delayed-detonation $\mathrm{N} 100$ model, the $\mathrm{W} 7$ model, and the solar-metallicity polluted $\mathrm{W} 7_{Z_{\odot}}$ model, respectively.

(A color version of this figure is available in the online journal.)

NIR sky flux was modeled using off-source pixels as described by Kelson (2003) and subtracted from the science frame before extraction, which was weighted by a simple boxcar profile. An A0V star was observed for telluric corrections, following the procedures outlined by Vacca et al. (2003). The xtellcor procedure, derived from the Spextool package (Cushing et al. 2004), performs both telluric correction and relative flux calibration. The corrected echelle orders are then combined into a single one-dimensional spectrum.

There is substantial wavelength overlap between the STIS and FIRE spectra (8295-10230 ̊). Using this overlap region, we scaled the FIRE spectrum to the STIS spectrum and combined the two spectra. The combined spectrum (1615-24880 $\AA$ ) is presented in Figures 2 and 3; it has been corrected for Milky Way reddening of $E(B-V)=0.011 \mathrm{mag}$ (Schlegel et al. 1998).

\section{RESULTS}

Our spectrum of SN 2011iv is the first high-S/N, nearmaximum, true-UV SN Ia spectrum since that of SN 1992A (K93), the earliest high-S/N UV SN Ia spectrum yet published, and the first contemporaneous and continuous UVOIR spectrum of an SN Ia. This unique data set provides the opportunity to examine the full SED, placing strong constraints on SN Ia models.

Fitting the $B$ and $V$ light curves (Figure 1) with a third-order polynomial function, we estimate that SN 2011 iv reached maximum light in the $B$ band on $2,455,906.0 \pm 0.3 \mathrm{JD}$ and peaked at $B=12.53 \pm 0.03 \mathrm{mag}$ and $V=12.51 \pm 0.03 \mathrm{mag}$. The $B_{\max }-V_{\max }$ pseudo-color is consistent with negligible reddening in the host galaxy. Assuming a Milky Way extinction of $A_{V}=0.038$ mag (Schlegel et al. 1998) and our preferred distance modulus, SN $2011 \mathrm{iv}$ peaked at $M_{V}=-19.06 \pm 0.08 \mathrm{mag}$. The optical light curves of SN 2011iv indicate that it is a relatively fast-declining SN with $\Delta m_{15}(B)=1.69 \pm 0.05$ mag; the light curves are well matched by the $\Delta m_{15}=1.69$ mag Hamuy et al. (1996) templates (Figure 1).

As seen in Figure 2, optical emission dominates the SEDs of SNe Ia at maximum light. The median flux over $100 \AA$ bins centered at 2500,3000 , and $3500 \AA$ is $2.4 \%, 18.1 \%$, and $50.0 \%$ that of the peak flux (in $f_{\lambda}$ units), respectively. Similarly, the median flux over $500 \AA$ bins centered at $1,1.5$, and $2 \mu \mathrm{m}$ is $3.6 \%$, $0.8 \%$, and $0.4 \%$ that of the peak flux (in $f_{\lambda}$ units), respectively.

SN 2011iv has a relatively strong silicon ratio, $\mathcal{R}(\mathrm{Si}$ II) (Nugent et al. 1995), of $0.50 \pm 0.05$. This measurement is consistent with its decline rate. Optical spectra of SN 2011iv are extremely similar to those of SNe 1992A (K93) and 2004eo (Pastorello et al. 2007). Both SNe 1992A and 2004eo also had relatively fast decline rates $\left(\Delta m_{15}(B)=1.48\right.$ and $1.46 \mathrm{mag}$, respectively), but were still spectroscopically "normal."

Using the method of Blondin et al. (2006), we determine the velocity of the SiII $\lambda 6355$ feature to be $v_{\text {Si II }}=$ $-10,630 \pm 130 \mathrm{~km} \mathrm{~s}^{-1}$. Using the relationships between $v_{\mathrm{Si} \text { II }}$ and maximum-light velocity, $v_{\mathrm{Si} \text { II }}^{0}$ (Foley et al. 2011), we derive 

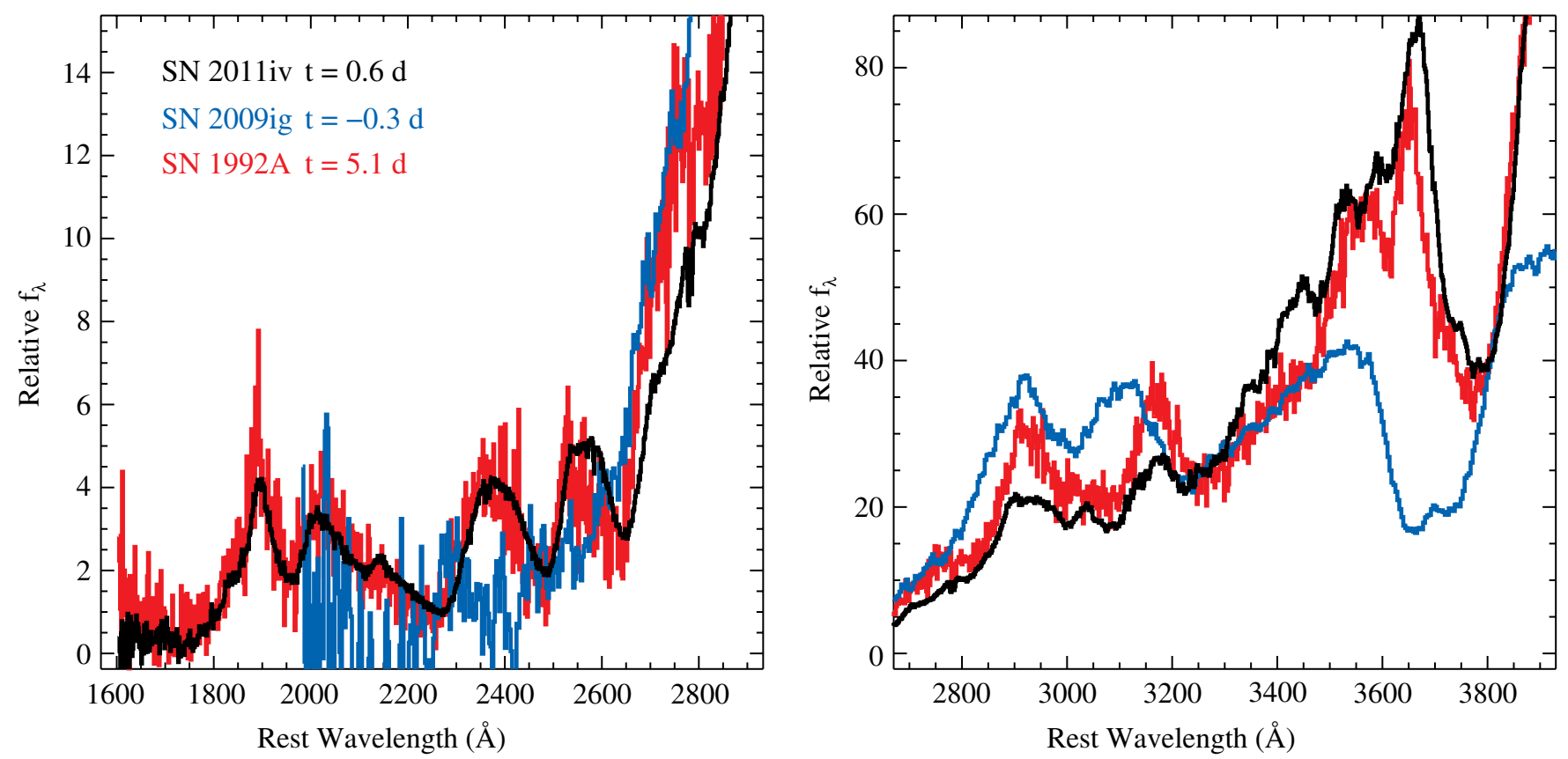

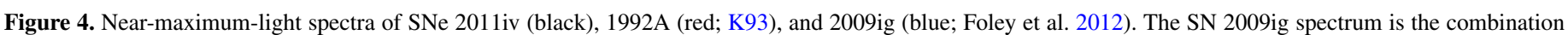
of the $t=-2.1$ and 1.5 day spectra (to increase the $\mathrm{S} / \mathrm{N}$ ). The spectra have been scaled to have similar flux at $3250 \AA$.

(A color version of this figure is available in the online journal.)

$v_{\mathrm{Si} \text { II }}^{0}=-10,700 \pm 300 \mathrm{~km} \mathrm{~s}^{-1}$; therefore, $v_{\mathrm{Si} \text { II }}^{0}$ of SN $2011 \mathrm{iv}$ is $\sim 800 \mathrm{~km} \mathrm{~s}^{-1}$ lower than the median value for the Foley et al. (2011) sample (it has a lower velocity than $84 \%$ of all SNe Ia in the sample).

\subsection{Comparison to Other Supernovae}

In Figure 4, we compare our SN 2011 iv spectrum to the nearmaximum UV spectra of SNe 1992A (K93) and 2009ig (Foley et al. 2012), which have both been dereddened by their Milky Way values of $E(B-V)=0.018$ and 0.032 mag (Schlegel et al. 1998), respectively. At $\lambda<3000 \AA$, the features in the SN 1992A and SN 2011iv spectra are remarkably similar, showing only minor differences. There is a slight wavelength offset in the features at 2300-2700 $\AA$, with the features being more blueshifted in the SN 1992A spectrum. Although SN 1992A was observed at an epoch 4.5 days later than SN 2011iv, SN 1992A had higher $v_{\mathrm{Si} \text { II }}^{0}$, and the higher ejecta velocity is likely the cause for the small differences in the UV. SN 2009ig is significantly different from the other two SNe; its spectrum lacks the prominent features at 2380 and $2560 \AA$ seen in both SN 1992A and SN 2011iv. There is perhaps an indication that the features are blueshifted even further than in SN 1992A, but the $\mathrm{S} / \mathrm{N}$ is too low to be conclusive. Similarly, the SN 2009ig spectrum does not have a sufficiently high $\mathrm{S} / \mathrm{N}$ at $\lambda<2300 \AA$ to say anything definitive about features in the far UV.

Examining the three SNe in the near-UV (2800-3800 ̊), there is even more diversity in their spectra. The spectra of SNe 1992A and 2011iv are still similar in this region, but SN 1992A has more pronounced features, especially surrounding Fe II $\lambda 3250$, which is observed at $\sim 3000 \AA$. Again, SN 2009ig has a very different spectrum from the other two SNe. Besides the clearly different $\mathrm{Ca} H \& \mathrm{~K}$ line profile, the biggest difference is in the continuum shape; SN 2009ig is relatively flat in the nearUV, while the other two SNe are much steeper. SN 2009ig has a very large $v_{\mathrm{Si} \text { II }}^{0}$, and the interpretation of Foley \& Kasen (2011) is that the large velocity range for absorbing lines, which correlates with $v_{\mathrm{Si} \text { II }}^{0}$, causes more line blanketing in the near-UV. Additional line blanketing, either from velocity or differences in the ejecta composition, may also explain the relatively flat spectral slope in the near-UV. Similar to what was seen with extremely low-resolution UV spectra (Wang et al. 2011), this sample of SNe Ia appears to have a large diversity in near-UV continua.

Over 2000-2500 $\AA$, SN 2011iv has an S/N of 28 per $1.5 \AA$ pixel, while SN 1992A has an S/N of 2.6 per $2 \AA$ pixel. The SN 2011iv spectrum has an $\mathrm{S} / \mathrm{N}$ that is $\sim 15$ times that of the SN 1992A spectrum. This exquisite spectrum shows additional features that are presumably hidden in the noise of the SN 1992A spectrum. Specifically, there is an absorption feature at $\sim 2120 \AA$ in the SN 2011iv spectrum that is not seen in the SN 1992A spectrum.

\subsection{Comparison to Models}

The majority of the SN luminosity is emitted at optical wavelengths. Most of this flux is redistributed from shorter wavelengths where IGEs provide significant line opacity due to millions of atomic line transitions. Although optical spectra are not particularly good at differentiating between models (Roepke et al. 2012), all models must reproduce the main optical signatures of SNe Ia to be credible.

It has been proposed that UV spectra of SNe Ia are a good tool to constrain different explosion models and progenitor metallicities (e.g., Höflich et al. 1998; Lentz et al. 2001). Our unique spectrum provides the possibility to test this assertion. For that purpose, we compare two different explosion models to our data. Specifically, we take the one-dimensional deflagration model W7 (Nomoto et al. 1984) and the state-of-the-art threedimensional delayed-detonation model N100 (Röpke et al., submitted). Both models yield $\sim 0.6 M_{\odot}$ of ${ }^{56} \mathrm{Ni}$, which is typical for normal SNe Ia (Stritzinger et al. 2006). For both models, we obtained a spectral time sequence with the Monte Carlo radiative transfer code ARTIS (Kromer \& Sim 2009). 
To study the influence of progenitor metallicity, we created an additional model $\mathrm{W} 7_{Z_{\odot}}$, in which we polluted the outer layers of W7 (zones where the sum of the IGE abundances was lower than solar IGE content, i.e., $v<-12,500 \mathrm{~km} \mathrm{~s}^{-1}$ ) with solar abundances. A comparison of maximum-light spectra of our three models and the UVOIR spectrum of SN 2011iv is shown in Figure 3. Both the $\mathrm{N} 100$ and $\mathrm{W} 7_{Z_{\odot}}$ models have similar SEDs to that of SN 2011iv from the UV to the NIR. In contrast, the original W7 model has excess UV flux.

The differences in the W7 spectra are mostly limited to the far-UV $(\lambda<2800 \AA)$, indicating that only this wavelength regime is strongly sensitive to progenitor metallicity (see also Lentz et al. 2001). If we want to use $\mathrm{SNe}$ Ia as distance indicators at $z \gtrsim 1.5$, for which the observed optical data correspond to the rest-frame far-UV, then light-curve fitting algorithms have to account for a range of different progenitor metallicities.

In contrast, our two metallicity points for W7 indicate that in the near-UV $(2800<\lambda<3800 \AA)$, the influence of different progenitor metallicities is weaker. This region might be better suited to differentiate between explosion models. Indeed, the N100 delayed-detonation model shows different characteristics than W7 in this regime and reproduces the near-UV features of SN 2011 iv somewhat better.

\section{DISCUSSION AND CONCLUSIONS}

We presented a UVOIR spectrum of SN 2011iv, a relatively normal SN Ia. These data were obtained 0.6 days after $B$-band maximum light with $H S T /$ STIS and Magellan/FIRE. This spectrum is the first contemporaneous and continuous UVOIR spectrum of an SN Ia. It is also the first published high-S/N, near-maximum, true-UV SN Ia spectrum since that of SN 1992A (K93) and the earliest high-S/N UV SN Ia spectrum yet published.

We compared the UV spectrum of SN 2011iv to the nearmaximum-light UV spectra of SNe 1992A and 2009ig. SNe 1992A and 2011iv have very similar UV spectra. However, SN 2009ig has a spectrum that is significantly different from that of the other two SNe. Specifically, SN 2009ig lacks two prominent features at 2300-2700 $\AA$ (though this may be the result of its higher ejecta velocity), and it has a relatively flat near-UV continuum.

Although the specific reasons for the differences between the UV spectra of the three SNe are not yet clear, the SNe have several additional observational differences. SNe 1992A, 2009ig, and 2011iv have S0, Sa, and E1 host galaxies; $\Delta m_{15}(B)=1.48$, 0.89 , and $1.69 \mathrm{mag}$; and $v_{\mathrm{Si} I}^{0}=-12,900,-13,500$, and $-10,700 \mathrm{~km} \mathrm{~s}^{-1}$, respectively (Foley et al. 2011, 2012). All three SNe have early-type host galaxies, so there is no clear difference in progenitor age or metallicity; additional SNe must be observed to probe the effects of environment on SN Ia UV spectra. Nonetheless, Sauer et al. (2008) were able to produce different near-UV continua (qualitatively similar to what is seen in Figure 4) by varying the amount of $\mathrm{Ti}$ and $\mathrm{Cr}$ generated in the explosion. Specifically, models with less $\mathrm{Ti} / \mathrm{Cr}$ had flatter near-UV continua.

SNe 1992A and 2009ig have similar $v_{\mathrm{Si} \text { II }}^{0}$, while SN 2011iv has significantly lower $v_{\mathrm{Si} \text { II }}^{0}$; however, SNe 1992A and 2009ig have different UV spectra, while SNe 1992A and 2011iv have very similar UV spectra. Finally, SNe 1992A and 2011iv are relatively fast-declining SNe Ia, while SN 2009ig is a relatively slow-declining SN Ia. It appears that the characteristics of SN Ia UV spectra are better predicted by light-curve shape than ejecta velocity. We hypothesize that ${ }^{56} \mathrm{Ni}$ generation, which regulates the decline rate (Kasen \& Woosley 2007), is dominant over the kinetic energy per unit mass in the formation of the UV spectra of SNe Ia near maximum light.

UV spectra are particularly sensitive to progenitor metallicity and the details of the explosion. The maximum-light spectrum of SN 2011iv is similar to both the delayed-detonation N100 model and the solar-metallicity polluted $\mathrm{W} 7_{Z_{\odot}}$ model spectra; the standard W7 model has significantly more far-UV flux than SN 2011iv. Additional data will be useful in determining the full density structure of the explosion.

This first spectrum shows the power of UV spectroscopy for understanding the progenitors and explosions of SNe Ia. Our full set of UV, optical, and NIR data for SN 2011iv, including seven $H S T$ spectra, will be analyzed in a later paper. SN 2011 iv is one of the best-observed SNe, and detailed analysis of this full data set should provide even stronger constraints on explosion models.

This research was supported by a Clay Fellowship (R.J.F.), an NSF Graduate Research Fellowship (E.R.N.), the TABASGO Foundation (A.V.F.), and NASA/HST grant GO-12592. G.P. acknowledges support by the Proyecto FONDECYT 11090421 , proyecto regular UNAB DI-28-11/R, and by the grant ICM P10-064-F (Millennium Center for Supernova Science), with input from the "Fondo de Innovaciṕn para la Competitividad, del Ministerio de Economía, Fomento y Turismo de Chile." S.T. is supported by the DFG (Transregional Collaborative Research Center TRR 33). The simulations were performed at JSC, Jülich, Germany (grants PRACE042, HMU14/20).

Facilities: HST (STIS), Magellan:Baade (FIRE), CTIO:PROMPT

\section{REFERENCES}

Blakeslee, J. P., Cantiello, M., Mei, S., et al. 2010, ApJ, 724, 657 Blondin, S., Dessart, L., Leibundgut, B., et al. 2006, AJ, 131, 1648 Bochanski, J. J., Hennawi, J. F., Simcoe, R. A., et al. 2009, PASP, 121, 1409

Bufano, F., Immler, S., Turatto, M., et al. 2009, ApJ, 700, 1456

Cooke, J., Ellis, R. S., Sullivan, M., et al. 2011, ApJ, 727, L35

Cushing, M. C., Vacca, W. D., \& Rayner, J. T. 2004, PASP, 116, 362

Drescher, C., \& Parker., 2011, CBET, 2940, 1

Foley, R. J. 2011, ATel, 3797, 1

Foley, R. J. 2012, ApJ, 748, 127

Foley, R. J., Challis, P. J., Filippenko, A. V., et al. 2012, ApJ, 744, 38

Foley, R. J., Filippenko, A. V., \& Jha, S. W. 2008, ApJ, 686, 117

Foley, R. J., \& Kasen, D. 2011, ApJ, 729, 55

Foley, R. J., Sanders, N. E., \& Kirshner, R. P. 2011, ApJ, 742, 89

Graham, A. W., Colless, M. M., Busarello, G., Zaggia, S., \& Longo, G. 1998, A\&AS, 133, 325

Hamuy, M., Phillips, M. M., Suntzeff, N. B., et al. 1996, AJ, 112, 2438

Hicken, M., Challis, P., Jha, S., et al. 2009, ApJ, 700, 331

Hillebrandt, W., \& Niemeyer, J. C. 2000, ARA\&A, 38, 191

Höflich, P., Wheeler, J. C., \& Thielemann, F.-K. 1998, ApJ, 495, 617

Howell, D. A., Sullivan, M., Conley, A., \& Carlberg, R. 2007, ApJ, 667, L37

Jha, S., Kirshner, R. P., Challis, P., et al. 2006, AJ, 131, 527

Kasen, D., \& Woosley, S. E. 2007, ApJ, 656, 661

Kelly, P. L., Hicken, M., Burke, D. L., Mandel, K. S., \& Kirshner, R. P. 2010, ApJ, 715,743

Kelson, D. D. 2003, PASP, 115, 688

Kirshner, R. P., Jeffery, D. J., Leibundgut, B., et al. 1993, ApJ, 415, 589

Kromer, M., \& Sim, S. A. 2009, MNRAS, 398, 1809

Lampeitl, H., Smith, M., Nichol, R. C., et al. 2010, ApJ, 722, 566

Lentz, E. J., Baron, E., Branch, D., \& Hauschildt, P. H. 2001, ApJ, 557, 266

Lentz, E. J., Baron, E., Branch, D., Hauschildt, P. H., \& Nugent, P. E. 2000, ApJ, 530,966 
Mazzali, P. A., Nomoto, K., Cappellaro, E., et al. 2001, ApJ, 547, 988 Mazzali, P. A., \& Podsiadlowski, P. 2006, MNRAS, 369, L19

Noguchi, T., Nakano, S., Chen, J., et al. 2011, CBET, 2940, 1

Nomoto, K., Thielemann, F.-K., \& Yokoi, K. 1984, ApJ, 286, 644

Nugent, P., Phillips, M., Baron, E., Branch, D., \& Hauschildt, P. 1995, ApJ, 455, L147

Pastorello, A., Mazzali, P. A., Pignata, G., et al. 2007, MNRAS, 377, 1531

Perlmutter, S., Aldering, G., Goldhaber, G., et al. 1999, ApJ, 517, 565

Phillips, M. M. 1993, ApJ, 413, L105

Reichart, D., Nysewander, M., Moran, J., et al. 2005, Nuovo Cimento C, 28, 767

Riess, A. G., Filippenko, A. V., Challis, P., et al. 1998, AJ, 116, 1009

Riess, A. G., Strolger, L.-G., Casertano, S., et al. 2007, ApJ, 659, 98
Roepke, F. K., Kromer, M., Seitenzahl, I. R., et al. 2012, ApJ, 750, L19 Sauer, D. N., Mazzali, P. A., Blondin, S., et al. 2008, MNRAS, 391, 1605 Schlegel, D. J., Finkbeiner, D. P., \& Davis, M. 1998, ApJ, 500, 525

Simcoe, R. A., Burgasser, A. J., Bernstein, R. A., et al. 2008, Proc. SPIE, 7014, 27

Stritzinger, M., Mazzali, P. A., Sollerman, J., \& Benetti, S. 2006, A\&A, 460, 793

Stritzinger, M., Morrell, N., \& Foley, R. J. 2011a, CBET, 2940, 1

Stritzinger, M. D., Phillips, M. M., Boldt, L. N., et al. 2011b, AJ, 142, 156

Sullivan, M., Conley, A., Howell, D. A., et al. 2010, MNRAS, 406, 782

Timmes, F. X., Brown, E. F., \& Truran, J. W. 2003, ApJ, 590, L83

Vacca, W. D., Cushing, M. C., \& Rayner, J. T. 2003, PASP, 115, 389

Wang, X., Wang, L., Filippenko, A. V., et al. 2011, ApJ, 749, 126 